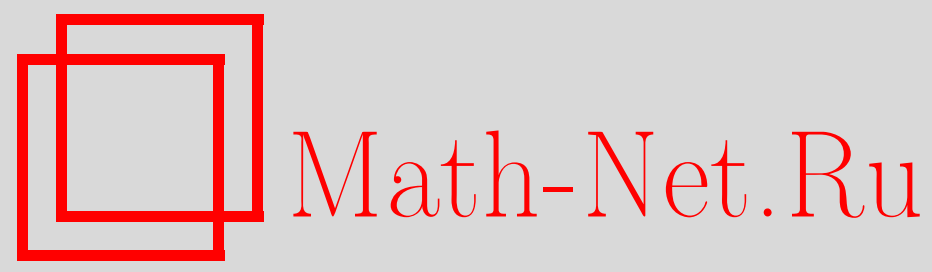

А. И. Астровский, Обобщенная матрица Грама и ее применение к проблеме наблюдаемости линейных нестационарных систем, Матем. заметки, 2001, том 69, выпуск 2, 163170

DOI: https://doi.org/10.4213/mzm492

Использование Общероссийского математического портала Math-Net.Ru подразумевает, что вы прочитали и согласны с пользовательским соглашением http://www.mathnet.ru/rus/agreement

Параметры загрузки:

IP: 54.84 .234 .179

26 апреля 2023 г., 14:45:00

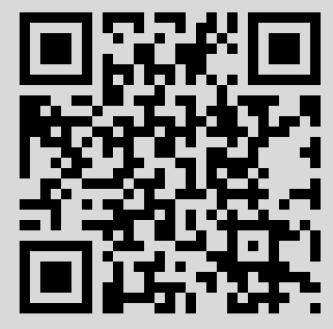


том 69 выпУСК 2 фЕВРАль 2001

\section{ОБОБЩЕННАЯ МАТРИЦА ГРАМА}

\section{И ЕЕ ПРИМЕНЕНИЕ К ПРОБЛЕМЕ НАБЛЮДАЕМОСТИ ЛИНЕЙНЫХ НЕСТАЦИОНАРНЫХ СИСТЕМ}

\section{А. И. Астровский}

Получены достаточные условия невырожденности обобщенной матрицы Грама. В частности, показано, что обобщенная матрица Грама невырождена для систем функций Чебышева. Дано применение полученных резульатов к задачам наблюдаемости линейных нестационарных систем обыкновенных дифференциальных уравнений. В терминах матрицы наблюдаемости доказаны необходимые и достаточные условия полной и тотальной наблюдаемости с помощью конечно-параметрических разрешающих операций.

Библиографояя: 21 название.

1. Обобщенная матрица Грама. Пусть дана $n$-вектор-функция-столбец $f(t)=$ $\left(f_{1}(t), f_{2}(t), \ldots, f_{n}(t)\right)$, элементы которой - вещественные кусочно-непрерьвные функции на отрезке $T=\left[t_{0}, t_{1}\right]$. Известно [1], что система функций $\left(f_{1}(t), f_{2}(t), \ldots, f_{n}(t)\right)$ линейно независима на $T$ тогда и только тогда, когда матрица

$$
G_{r}\left(f, t_{0}, t_{1}\right)=\int_{t_{0}}^{t_{1}} f(t) f^{\prime}(t) d t
$$

невырождена. Штрих означает операцию транспонирования.

При исследовании задач управления в специальных классах управляющих воздействий, а также задач наблюдения с помощью специальных классов разрешаюших операций [2] возникает необходимость изучения матрицы, построенной следующим образом.

Пусть на отрезке $\left[t_{0}, t_{1}\right]$ заданы две кусочно-непрерывные $n$-вектор-функции-столбцы

$$
f(t)=\left(f_{1}(t), f_{2}(t), \ldots, f_{n}(t)\right), \quad h(t)=\left(h_{1}(t), h_{2}(t), \ldots, h_{n}(t)\right) .
$$

Определим матрицу $G\left(f, h, t_{0}, \tau\right), \tau \in T$, следуюшим образом:

$$
G\left(f, h, t_{0}, \tau\right)=\int_{t_{0}}^{\tau} f(t) h^{\prime}(t) d t .
$$

В дальнейшем матрицу $G\left(f, h, t_{0}, \tau\right)$ вида (3) будем назьвать обобщенной матричей Грама.

Рассматривается следующая задача: найти условия, при которых существует такой момент $\tau^{*} \in T$, что матрица $G\left(f, h, t_{0}, \tau^{*}\right)$ невырождена. 
Ясно, что для существования момента $\tau^{*} \in T$, при котором определитель $\operatorname{det} G(f, h$, $\left.t_{0}, \tau^{*}\right)$ матрицы $G\left(f, h, t_{0}, \tau^{*}\right)$ отличен от нуля, необходимо, чтобы как система функций $f(t)=\left(f_{1}(t), f_{2}(t), \ldots, f_{n}(t)\right)$, так и система функций $h(t)=\left(h_{1}(t), h_{2}(t), \ldots, h_{n}(t)\right)$ были линейно независимы на $\left[t_{0}, \tau^{*}\right]$.

Несложно привести пример того, что условия линейной независимости системы функций $f(t)$ и линейной независимости системы функций $h(t)$ на $T$ не являются достаточными условиями невырожденности матрищы $G\left(f, h, t_{0}, \tau^{*}\right)$ хотя бы при одном $\tau^{*} \in T$.

Если $n$-вектор-функции $f(t)$ и $h(t)$ таковы, что $f(t)=P h(t), t \in T$, для некоторой постоянной матрицы $P$, то $G\left(f, h, t_{0}, \tau\right)=P G_{r}\left(h, t_{0}, \tau\right), \tau \in T$, и при $\operatorname{det} P \neq 0$ матрица $G\left(f, h, t_{0}, \tau\right)$ невырождена тогда и только тогда, когда невырождена матрица $G_{r}\left(f, t_{0}, \tau\right), \tau \in\left(t_{0}, t_{1}\right]$.

Отметим, что при изучении задач управляемости для линейных стационарных систем обыкновенных дифференциальных уравнений в специальных классах управлений невырожденность матрицы вида (3) исследовалась в [3]-[6] для специального вида функций $f(t)$ и конкретно заданных функций $h(t)$. При этом исследование невырожденности матрицы $G\left(f, h, t_{0}, \tau\right)$ проводилось на основе разложения определителя этой матрищы в ряд Тейлора в окрестности точки $t_{0}$, для чего приходилось дифференцировать $n^{2}$ раз определитель матрищы $G\left(f, h, t_{0}, \tau\right), \tau \in T$. В [7], [8] при изучении задач наблюдения с помощью специальных классов разрешающих операций для нестационарных линейных систем была рассмотрена обобщенная матрица Грама для систем функций Чебьшева (T-систем).

Напомним, что система вещественных непрерьвных функций $\left(h_{1}(t), h_{2}(t), \ldots, h_{n}(t)\right)$ назьвается [9], [10] системой функиий Чебышева порядка $n-1$ на $T$, если любая нетривиальная линейная комбинация этих функций имеет не более $n-1$ корня на $T$. Для случая, когда компоненты $n$-вектор-функций $f$ и $h$ являются системами функций Чебышева, доказано [7], что матрица $G\left(f, h, t_{0}, \tau^{*}\right)$ невырождена для любого $\tau^{*} \in\left(t_{0}, t_{1}\right]$. Доказательство этого утверждения основьвалось на использовании свойств $C-J$ докритичности из [11]. Отметим также, что результаты работ [4]-[6] следуют из [7], [8] при специально заданных системах функций $\left(h_{1}(t), h_{2}(t), \ldots, h_{n}(t)\right)$, например для функций, порождающих полиномы степени $n$, тригонометрические полиномы и т.д.

Ниже приводится доказательство невырожденности обобщенной матрицы Грама, основанное исключительно на свойствах систем функций Чебьшева.

Теорема 1. Пусть даны две системы функиий Чебышева $f(t)=\left(f_{1}(t), f_{2}(t), \ldots\right.$, $\left.f_{n}(t)\right) u h(t)=\left(h_{1}(t), h_{2}(t), \ldots, h_{n}(t)\right)$ порядка $n-1$ на $\left[t_{0}, t_{1}\right]$. Тогда $\operatorname{det} G\left(f, h, t_{0}\right.$, $\left.\tau^{*}\right) \neq 0$ для любого $\tau^{*} \in\left(t_{0}, t_{1}\right]$.

ДокАЗАТЕЛЬСтво. Пусть существует такой момент $\tau^{*} \in\left(t_{0}, t_{1}\right]$, что матрица $G(f$, $\left.h, t_{0}, \tau^{*}\right)$ вырождена. Это означает, что найдется такой ненулевой вектор $g=\left(g_{1}, g_{2}, \ldots\right.$, $\left.g_{n}\right) \in \mathbb{R}^{n}$, что $G\left(f, h, t_{0}, \tau^{*}\right) g=0$. Поскольку $\left(h_{1}(t), h_{2}(t), \ldots, h_{n}(t)\right)$ является системой функций Чебьшшева порядка $n-1$ на $\left[t_{0}, t_{1}\right]$, то функция $\omega_{h}(t)=h_{1}(t) g_{1}+h_{2}(t) g_{2}+\cdots$ $\cdots+h_{n}(t) g_{n}$ имеет не более $n-1$ корня на $T$. Обозначим неузловые корни функции $\omega_{h}(t), t \in T$, через $\tau_{1}, \tau_{2}, \ldots, \tau_{k}$, а корни-узлы - через $\tau_{k+1}, \tau_{k+2}, \ldots, \tau_{k+l}$. Из свойств $T$-систем следует [9], [10], что $2 k+l \leqslant n-1$. Поэтому среди многочленов по системе функций Чебьшева $\left(f_{1}(t), f_{2}(t), \ldots, f_{n}(t)\right)$ существует [10] такой многочлен $\omega_{f}(t)=\sum_{i=1}^{n} \alpha_{i} f_{i}(t)$, что точки $\tau_{1}, \tau_{2}, \ldots, \tau_{k}$ являются неузловьми корнями, а точки $\tau_{k+1}, \tau_{k+2}, \ldots, \tau_{k+l}$ - корнями-узлами многочлена $\omega_{f}(t)$, причем у функции $\omega_{f}(t), t \in T$, других корней нет. Отметим, что на отрезках $\left[\tau_{j}, \tau_{j+1}\right], j \in(1,2, \ldots, k+l-1)$, функции $\omega_{f}(t)$ и $\omega_{h}(t)$ имеют один и тот же знак. Следовательно, справедливо неравенство 
$\omega_{h}(t) \omega_{f}(t) \geqslant 0, t \in T$, причем равенство нулю будет только в точках $\tau_{j}$. Обозначим через $\alpha n$-вектор-строку $\left(\alpha_{1}, \ldots, \alpha_{n}\right)$. Из равенства

$$
\alpha G\left(f, h, t_{0}, \tau^{*}\right) g=\int_{t_{0}}^{\tau^{*}} \omega_{h}(t) \omega_{f}(t) d t=0
$$

получим тождество $\omega_{h}(t) \omega_{f}(t) \equiv 0, t \in\left[t_{0}, \tau^{*}\right]$, которое противоречит соотношению $\omega_{h}(t) \omega_{f}(t) \geqslant 0, t \in T$. Теорема доказана.

Пусть функции $f_{i}(t)$ и $h_{i}(t), i \in(1,2, \ldots, n)$, определены и непрерывно дифференцируемы $n-1$ раз на открытом множестве $\Delta$, содержащем отрезок $T$. Для $k \in(1,2, \ldots, n)$ обозначим через $W_{f}^{k}(t)$ определитель Вронского системы функций $\left(f_{1}(t), f_{2}(t), \ldots\right.$, $\left.f_{k}(t)\right)$.

Лемма 1. Если существует такая точка $\tau^{0} \in\left[t_{0}, t_{1}\right]$, в окрестности которой функиии $f_{i}(t)$ u $h_{i}(t), i \in(1,2, \ldots, n)$, непрерывно дифференцируемы $n-1$ раз и

$$
W_{f}^{n}\left(\tau^{0}\right) \neq 0, \quad W_{h}^{n}\left(\tau^{0}\right) \neq 0
$$

то найдется такой отрезок $\left[\xi_{0}, \xi_{1}\right] \subseteq\left[t_{0}, t_{1}\right]$, что $\tau^{0} \in\left[\xi_{0}, \xi_{1}\right]$ и матрииа $G\left(f, h, \xi_{0}\right.$, $\tau)$ невырохсдена для любого $\tau \in\left(\xi_{0}, \xi_{1}\right]$.

ДокАЗАТЕльство. Пусть условие (4) вьполняется. Тогда существует [12], [13] такой отрезок $\left[\xi_{0}, \xi_{1}\right] \subseteq\left[t_{0}, t_{1}\right], \tau^{0} \in\left[\xi_{0}, \xi_{1}\right]$, на котором функции $\left(f_{1}(t), f_{2}(t), \ldots, f_{n}(t)\right)$ и $\left(h_{1}(t), h_{2}(t), \ldots, h_{n}(t)\right)$ образуют системы функций Чебышева порядка $n-1$. В силу теоремы $1 \operatorname{det} G\left(f, h, \xi_{0}, \tau\right) \neq 0$ для любой точки $\tau \in\left(\xi_{0}, \xi_{1}\right]$. Лемма доказана.

Условия леммы 1 гарантируют существование такого отрезка $\left[\xi_{0}, \xi_{1}\right] \subseteq\left[t_{0}, t_{1}\right]$, что обобщенная матрища Грама $G\left(f, h, \xi_{0}, \tau\right)$ невырождена для любого $\tau \in\left(\xi_{0}, \xi_{1}\right]$. Следующее утверждение дает условия невырожденности обобщенной матрицы Грама на заданном отрезке.

Лемма 2. Если на интервале $\left(t_{0}, t_{1}\right)$ функиии $\left(f_{1}(t), f_{2}(t), \ldots, f_{n}(t)\right) u\left(h_{1}(t)\right.$, $\left.h_{2}(t), \ldots, h_{n}(t)\right)$ непрерывно дифференчируемы $(n-1)$ раз и при кажсом $k \in(1,2$, $\ldots, n)$ выполняются условия

$$
W_{f}^{k}(\tau) \neq 0, \quad W_{h}^{k}(\tau) \neq 0 \quad \text { для любого } \tau \in\left(t_{0}, t_{1}\right)
$$

то обобщенная матрица Грама $G\left(f, h, t_{0}, \tau\right)$ невырождена при всех $\tau \in\left(t_{0}, t_{1}\right)$.

ДоКАЗАТЕЛЬСТВо. Из [13], [14] следует, что при вьполнении условия (5) любые линейные комбинации $\alpha_{1} f_{1}(t)+\alpha_{2} f_{2}(t)+\cdots+\alpha_{n} f_{n}(t)$ и $\alpha_{1} h_{1}(t)+\alpha_{2} h_{2}(t)+\cdots+\alpha_{n} h_{n}(t)$ с вещественньми $\alpha_{1}, \alpha_{2}, \ldots, \alpha_{n}$, в совокупности отличными от нуля, имеют не более $n-1$ нулей на $\left(t_{0}, t_{1}\right)$. Следовательно, системы функций $\left(f_{1}(t), f_{2}(t), \ldots, f_{n}(t)\right)$ и $\left(h_{1}(t), h_{2}(t)\right.$, $\left.\ldots, h_{n}(t)\right)$ являются системами функций Чебьшева порядка $n-1$ на $\left(t_{0}, t_{1}\right)$. Поэтому в силу теоремы 1 обобщенная матрица Грама $G\left(f, h, t_{0}, \tau\right)$ невырождена при любом $\tau \in\left(t_{0}, t_{1}\right)$. 
ЗАмЕчАНИЕ 1. При исследовании обобщенной матрицы Грама полезным оказьвается понятие раствора $\theta\left(H_{1}, H_{2}\right)$ [15] между подпространствами $H_{1}$ и $H_{2}$ гильбертова пространства $H$. Пусть $L_{2}\left(t_{0}, \tau\right)$ - гильбертово пространство действительных функций, квадрат которых интегрируем по Лебегу на отрезке $\left[t_{0}, \tau\right], t_{0}<\tau \leqslant t_{1}$, со скалярным произведением

$$
(a, b)=\int_{t_{0}}^{\tau} a(t) b(t) d t, \quad a, b \in L_{2}\left(t_{0}, \tau\right) .
$$

Через $H_{f}(\tau)$ и $H_{h}(\tau)$ обозначим подпространства из $L_{2}\left(t_{0}, \tau\right)$, порожденные соответственно системами функций $f(t)$ и $h(t), t \in\left[t_{0}, \tau\right]$. Если существует такой момент $\tau^{*} \in\left(t_{0}, t_{1}\right]$, при котором раствор $\theta\left(H_{f}\left(\tau^{*}\right), H_{h}\left(\tau^{*}\right)\right)<1$, то [15] обобщенная матрица Грама $G\left(f, h, t_{0}, \tau^{*}\right)$ невырождена.

2. Наблюдаемость с помощью конечно-параметрических разрешающих операций. Рассмотрим линейную нестационарную систему обькновенных дифференциальных уравнений

$$
\dot{x}(t)=A(t) x(t), \quad t \in T=\left[t_{0}, t_{1}\right] .
$$

Здесь $x(t)-n$-вектор-столбец состояния системы (6) в момент $t$, a $(n \times n)$-матрица $A(t)$ является непрерывной матричной функцией.

Предположим, что выходная функция $y(t)=y\left(t, x_{0}\right)$ системы (6) зависит от ее состояния $x(t)=x\left(t, x_{0}\right)$ в момент $t$ следующим образом:

$$
y\left(t, x_{0}\right)=c(t) x(t), \quad t \in T,
$$

где $c(t)-n$-вектор-функция-строка с непрерывными элементами; $x_{0}=x\left(t_{0}\right) \in \mathbb{R}^{n}-$ начальное состояние системы (6).

Пусть $F(t)$ - фундаментальная матрица системы (6):

$$
\dot{F}(t)=A(t) F(t), \quad F\left(t_{0}\right)=E, \quad t \in T .
$$

Здесь $E$ - единичная $(n \times n)$-матрица.

Обозначим через $C(T, \mathbb{R})$ множество непрерывных скалярных функций на отрезке $T$, а через $\mathscr{Y} \subset C(T, \mathbb{R})$ - совокупность всех вьходных функций системы $(6),(7)$ :

$$
\mathscr{Y}=\left\{y \in C(T, \mathbb{R}): y=y\left(x_{0}\right)=\left(y\left(t, x_{0}\right)=h(t) x_{0}, t \in T\right), \quad x_{0} \in \mathbb{R}^{n}\right\},
$$

где $n$-вектор-функция-строка $h(t)=\left(h_{1}(t), h_{2}(t), \ldots, h_{n}(t)\right)$ равна $c(t) F(t), t \in T$.

ОПРЕДЕЛЕНИЕ 1 [2], [16]. Система (6), (7) называется полностью наблюдаемой на $T$, если отображение множества начальных состояний $\mathbb{R}^{n}$ в множество выходных функций У системы (6), (7) взаимно однозначно.

Так как система наблюдения (6), (7) задает отображение $\Psi: \mathbb{R}^{n} \rightarrow \mathscr{Y}$ по правилу: $y\left(t, x_{0}\right)=h(t) x_{0}, t \in T$, то свойство полной наблюдаемости системы (6), (7) эквивалентно инъективности оператора $\Psi$. Последнее равносильно линейной независимости системы функций $\left(h_{1}(t), h_{2}(t), \ldots, h_{n}(t)\right)$ на отрезке $T$.

Как следует из [2], полная наблюдаемость системы (6), (7) гарантирует для каждого $p \in \mathbb{R}^{n}$ существование такой измеримой почти всюду ограниченной функции $r$, что

$$
\int_{t_{0}}^{t_{1}} r(t) y\left(t, x_{0}\right) d t=p^{\prime} x_{0}
$$


для всех $x_{0} \in \mathbb{R}^{n}$. Функционалы

$$
\varphi\left[y\left(x_{0}\right)\right]=\int_{t_{0}}^{t_{1}} r(t) y\left(t, x_{0}\right) d t
$$

удовлетворяюшие соотношению (8), назьваются разрешающими операциями [2], а функции $r$, их порождающие, также называют разрешающ,ими.

С точки зрения построения разрешающих операций представляет интерес существование разрешающих функций в классе более узком, чем класс измеримых почти всюду ограниченных функций. В литературе исследовалось существование разрешаюших операций при ограничениях на разрешающие функции как геометрического и интегрального характера (т.е. на область возможных значений), так и при функциональных ограничениях [2], [3], [7], [8], [16], [17] и т.д.

Итак, пусть на $T$ задано некоторое множество скалярных функций $\mathscr{F}$.

ОПРЕДЕЛЕНИЕ 2. Система (6), (7) наблюдаема в классе разрешающих функиий $\mathscr{F}$ (кратко: $\mathscr{F}$-наблюдаема), если существует такой момент $\tau^{*} \in\left(t_{0}, t_{1}\right]$, что для каждого $p \in \mathbb{R}^{n}$ найдется функция $r_{p} \in \mathscr{F}$, для которой справедливо равенство

$$
\int_{t_{0}}^{\tau^{*}} r_{p}(t) y\left(t, x_{0}\right) d t=p^{\prime} x_{0}
$$

для всех $x_{0} \in \mathbb{R}^{n}$.

Ниже рассмотрим задачу существования разрешающих функций из класса конечно-параметрических функций, т.е. из класса функций $\mathscr{F}$ следующего вида:

$$
\mathscr{F}=\left\{r(\cdot): r(t)=\alpha_{1} \gamma_{1}(t)+\cdots+\alpha_{n} \gamma_{n}(t), \quad \alpha=\left(\alpha_{1}, \ldots, \alpha_{n}\right)^{\prime} \in \mathbb{R}^{n}, \quad t \in T\right\},
$$

где $\gamma(t)=\left(\gamma_{1}(t), \gamma_{2}(t), \ldots, \gamma_{n}(t)\right)$ - заданная $n$-вектор-функция-столбец, компоненты которой кусочно-непрерьвны и линейно независимы на $T$.

Задачу $\mathscr{F}$-наблюдаемости системы (6), (7) с помощью конечно-параметрических функций вида (9) можно интерпретировать как задачу наблюдаемости системы (6), (7) с помощью динамического фильтра в следующей постановке.

Пусть известно, что выходная функция $y\left(t, x_{0}\right), t \in T$, системы $(6),(7)$ подается на вход линейной нестационарной системы обыкновенных дифференциальных уравнений (динамического фильтра)

$$
\dot{z}(t)=D(t) z(t)+b(t) y\left(t, x_{0}\right), \quad z\left(t_{0}\right)=0, \quad t \in T,
$$

где $z(t)=z\left(t, x_{0}\right)-n$-вектор состояния динамического фильтра (10) в момент $t$, порожденньй начальным состоянием $x\left(t_{0}\right)=x_{0} \in \mathbb{R}^{n}$ системы $(6),(7) ; D(t), b(t)$ - заданные соответственно $(n \times n)$-, $(n \times 1)$-матричные функции с непрерьвньми элементами.

ОПРЕДЕЛЕНИЕ 3 [17]. Система (6), (7) назьвается наблюдаемой с помощъю динамического фильтра (10), если существует такой момент $t^{*} \in\left(t_{0}, t_{1}\right]$, что для каждого $p \in \mathbb{R}^{n}$ найдется вектор $\alpha \in \mathbb{R}^{n}$, для которого справедливо равенство $\alpha^{\prime} z\left(t^{*}, x_{0}\right)=p^{\prime} x_{0}$ при любом $x_{0} \in \mathbb{R}^{n}$.

Из определения 3 следует, что для того чтобы определить начальное состояние системы (6) с помощью динамического фильтра (10) достаточно знать значение $n$-вектора 
$z\left(t, x_{0}\right)$ лишь в одной точке $t^{*}$, тогда как классическая задача наблюдения требует знания функции $y(t)$ на некотором отрезке времени (либо в совокупности точек из отрезка наблюдения).

Нетрудно заметить, что система (6), (7) наблюдаема с помощью динамического фильтра (10) тогда и только тогда, когда она наблюдаема в классе конечно-параметрических разрешающих функций следующего вида:

$$
\mathscr{F}_{1}=\left\{r(\cdot): r(t)=\alpha^{\prime} F_{1}^{-1}(t) b(t), \quad \alpha \in \mathbb{R}^{n}, \quad t \in T\right\},
$$

где $F_{1}(t)$ - фундаментальная матрица системы $\dot{z}(t)=D(t) z(t)$.

Непосредственно из теории линейных уравнений в конечномерных пространствах следует справедливость утверждения.

Лемма 3. Для $\mathscr{F - н а б л ю д а е м о с т и ~ с и с т е м ы ~ ( 6 ) , ~ ( 7 ) ~ в ~ к л а с с е ~ ф у н к и и и ̆ ~ ( 9 ) ~ н е - ~}$ обходимо и достаточно, чтобы существовал такой момент $\tau^{*} \in\left(t_{0}, t_{1}\right]$, что обобщенная матрица Грама $G\left(\gamma, h, t_{0}, \tau^{*}\right)=\int_{t_{0}}^{\tau^{*}} \gamma(t) h(t) d t$ невырождена.

Из леммы 3 следует

Лемма 4. Для भ्F-наблюдаемости системы (6), (7) достаточно, чтобы существовал такой момент $\tau^{*} \in\left(t_{0}, t_{1}\right]$, что раствор между линейными оболочками $H_{\gamma}\left(\tau^{*}\right)$ и $H_{h}\left(\tau^{*}\right)$ систем функиий $\left(\gamma_{1}(t), \gamma_{2}(t), \ldots, \gamma_{n}(t)\right) u\left(h_{1}(t), h_{2}(t), \ldots, h_{n}(t)\right)$, $t \in\left[t_{0}, \tau^{*}\right]$, удовлетворяет условию $\theta\left(H_{\gamma}\left(\tau^{*}\right), H_{h}\left(\tau^{*}\right)\right)<1$.

Так как получить в явном виде фундаментальную матрицу $F(t)$ для нестационарной системы (6) удается лишь в редких, исключительных случаях, то возникает задача исследования проблемы наблюдаемости без использования явного вида фундаментальной матрицы $F(t)$. Выразить условия наблюдаемости непосредственно через параметры исходной системы наблюдения удается с помощью матрицы наблюдаемости [2], [18], [19], построенной для достаточно гладких систем. Более того, матрицы наблюдаемости применяются при исследовании структурных свойств систем и полезны при нахождении преобразований, приводящих системы наблюдения к каноническим формам.

Ниже приводится схема построения матрицы наблюдаемости [20] для систем наблюдения $(6),(7)$. Для этого отождествим систему $(6),(7)$ с парой $(A, c)$, состоящей из матричной функции $A(t)$ и векторной функции-строки $c(t)$. Множество всех таких пар с непрерьвными компонентами обозначим $\Sigma$. Рассмотрим оператор [20] $L: \Sigma \rightarrow \Sigma$, действующий по правилу $L(A, c)=(A, c A+\dot{c})$. Область определения $\operatorname{dom}(L)$ оператора $L$ состоит из таких пар $(A, c)$, у которых функция $c(t)$ непрерьвно дифференцируема. Если компоненты $n$-вектор-функции-строки $s(t)=c(t) A(t)+\dot{c}(t)$ принадлежат классу $C^{1}$, то на паре $(A, c)$ определен оператор $L^{2}$, для которого $L^{2}(A, c)=L(A, s)$. По индукции можно определить любую степень $L^{k}$ оператора $L$. Обозначим $\operatorname{dom}\left(L^{k}\right)$ область определения оператора $L^{k}$. Для $k=0$ полагаем $L^{0}(A, c)=(A, c)$ и $\operatorname{dom}\left(L^{0}\right)=\Sigma$. Каждую систему $(A, c) \in \operatorname{dom}\left(L^{k}\right)$ назовем системой класса $k$. Отметим, что если компоненты матричной функции $A(t)$ принадлежат классу $C^{k-1}$, а компоненты векторной функции $c(t)$ принадлежат классу $C^{k}$, то $(A, c) \in \operatorname{dom}\left(L^{k}\right)$. Обратное, вообще говоря, не верно. В [20] приведен ряд утверждений, касающихся систем класса $k$.

Пусть система $(A, c) \in \Sigma$ имеет класс $n-1$. Тогда определены векторные функции строки

$$
s_{i}(t)=s_{i-1}(t) A(t)+\dot{s}_{i-1}(t), \quad s_{0}(t)=c(t), \quad i \in(1,2, \ldots, n-1),
$$


из которых составим $(n \times n)$-матрицу наблюдаемости

$$
S(t)=\left(s_{i}(t)\right)_{i=\overline{0, n-1}} .
$$

Отметим, что если система (6), (7) $\mathscr{F}$-наблюдаема, то она является полностью наблюдаемой и поэтому необходимые условия полной наблюдаемости (см., например, [2], $[16],[17],[19])$ являются и необходимыми условиями $\mathscr{F}$-наблюдаемости.

Далее исследуем задачу $\mathscr{F}$-наблюдаемости для случая, когда система функций $\gamma(t)=$ $\left(\gamma_{1}(t), \gamma_{2}(t), \ldots, \gamma_{n}(t)\right)$, которая определяет в $(9)$ класс функций $\mathscr{F}$, является системой функций Чебьшева порядка $n-1$ на отрезке $\left[t_{0}, t_{1}\right]$.

Из [7], [8] следует, что если $\operatorname{rank} S\left(t_{0}\right)=n$, то существует такой отрезок $\left[t_{0}, \tau_{1}\right]$, на котором функции $\left(h_{1}(t), h_{2}(t), \ldots, h_{n}(t)\right)=c(t) F(t)$ образуют систему функций Чебышева порядка $(n-1)$.

Из вьшеизложенного и результатов работы [7] следует справедливость утверждения.

Теорема 2. Для $\mathscr{F - н а б л ю д а е м о с т и ~ п а р ы ~}(A, c)$ класса $n-1$ с помощью многочленов систем функиий Чебышева достаточно, чтобы $\operatorname{rank} S\left(t_{0}\right)=n$.

Получим условия наблюдаемости системы (6), (7) с помощью динамического фильтра (10). Обозначим для пары $(D, b)$ класса $n-1$ через $Q(t)$ ее матрицу управляемости:

$$
Q(t)=\left(q_{i}(t)\right)_{i=\overline{0, n-1}}, \quad t \in T
$$

где $n$-вектор-столбцы $q_{i}(t)$ определяются по реккурентной формуле

$$
q_{i}(t)=D(t) q_{i-1}(t)-\dot{q}_{i-1}(t), \quad q_{0}(t)=b(t), \quad i \in(1,2, \ldots, n-1) .
$$

ТЕОрема 3. Пусть система (6), (7) и динамический фильтр (10) являются системами класса $n-1$ и выполняются условия: $\operatorname{rank} Q\left(t_{0}\right)=n, \operatorname{rank} S\left(t_{0}\right)=n$. Тогда система (6), (7) наблюдаема с помощью динамического фильтра (10).

Доказательство теоремы 3 основано на теореме 2 и представлении динамического фильтра через конечно-параметрические разрешаюшие операции.

3. Тотальная наблюдаемость. Хорошо известно, что если линейная стационарная система обыкновенных дифференциальных уравнений наблюдаема на отрезке $T=$ $\left[t_{0}, t_{1}\right]$, то она наблюдаема и на любом подотрезке из $T$. Для линейной нестационарной системы это утверждение неверно. В связи с этим в работах [18], [21] были введены и исследованы понятия тотальной (дифференциальной) наблюдаемости и управляемости. Ниже рассмотрим тотальную наблюдаемость с помощью конечно-параметрических разрешающих функций вида (9).

ОПРЕДЕЛЕНИЕ 4. Система (6), (7) тотально наблюдаема в классе разрешающих

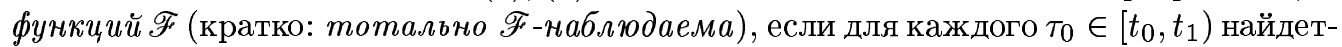
ся такой момент $\tau^{*} \in\left(t_{0}, t_{1}\right]$, что для любого $p \in \mathbb{R}^{n}$ сушествует функция $r_{p} \in \mathscr{F}$ такая, что для всякого $x_{0}=x\left(\tau_{0}\right) \in \mathbb{R}^{n}$ справедливо равенство

$$
\int_{\tau_{0}}^{\tau^{*}} r_{p}(t) y\left(t, x_{0}\right) d t=p^{\prime} x_{0}
$$


Лемма 5. Для тотальной $\mathscr{F - н а б л ю д а е м о с т и ~ с и с т е м ы ~ ( 6 ) , ~ ( 7 ) ~ в ~ к л а с с е ~ ф у н к - ~}$ иий (9) необходимо и достаточно, чтобы каждому $\tau_{0} \in\left[t_{0}, t_{1}\right)$ отвечал такой момент $\tau^{*} \in\left(t_{0}, t_{1}\right]$, что обобщенная матрича Грама $G\left(\gamma, h, \tau_{0}, \tau^{*}\right)$ невырохдена.

Из лемм 1 и 5 следует справедливость теоремы.

Теорема 4. Для тотальной $\mathscr{F - н а б л ю д а е м о с т и ~ п а р ы ~}(A, c)$ класса $n-1$ с помощью многочленов систем функиий Чебышева необходимо и достаточно, чтобь $\operatorname{rank} S(t)=n$ для почти всех $t \in T$.

Считаю своим приятным долгом выразить признательность рецензенту за полезные редакционные замечания.

\section{СПИСОК ЦИТИРОВАННОЙ ЛИТЕРАТУРЫ}

[1] Гантмахер Ф.Р. Теория матриц. М.: Наука, 1988.

[2] Красовский Н. Н. Теория управления движением. М.: Наука, 1968.

[3] Габасов Р., Кириллова Ф. М. Качественная теория оптимальных процессов. М.: Наука, 1971.

[4] Трох И. Замечания о $n$-наблюдаемости и $n$-управляемости // Механика. Сб. переводов иностр. статей. 1974. Т. 146. № 4. С. 255-264.

[5] Шкляр Б. Ш. Об управляемости в классах простейших функций // Вестн. БГУ. Сер. 1. 1972. № 1. C. 91-93.

[6] Игнатенко В.В. Управляемость динамических систем с помощью регулятора // Вестн. БГУ. Сер. 1. 1976. №2. С. 56-58.

[7] Астровский А.И. Наблюдаемость нестационарных линейных систем // Препринт ИМ АН Беларуси. 1978. № 8(40).

[8] Астровский А. И. Исследования по теории многосвязн. систем. М.: Наука, 1982.

[9] Крейн М. Г., Нудельман А. А. Проблема моментов Маркова и экстремальные задачи. М.: Наука, 1973.

[10] Карлин С., Стадден В. Чебышевские системы и их применение в анализе и статистике. М.: Наука, 1976.

[11] Тонков Е.Л. Неосцилляция и число переключений в линейной нестационарной системе, оптимальной по быстродействию // Дифференц. уравнения. 1973. Т. 9. №12. С. 2180-2185.

[12] Бернштейн С. Н. Экстремальные свойства полиномов. Л.-М.: ОНТИ, 1937.

[13] Vallée-Poussin C. Sur l'équation differentielle lineaire du second ordre // J. Math. Pure Appl. (9). 1928. V. 8. P. 125-144.

[14] Спринджук В. Г. Метод тригонометрических сумм в метрической теории диофантовых приближений зависимых величин // Тр. МИАН. 1972. Т. 128. С. 212-228.

[15] Красносельский М. А., Вайникко Г. М., Забрейко П. П., Рутицкий Я. Б., Стеценко В.Я. Приближенное решение операторных уравнений. М.: Наука, 1969.

[16] Куржанский А. Б. Управление и наблюдение в условиях неопределенности. М.: Наука, 1977.

[17] Астровский А. И., Мулярчик В.В.О наблюдаемости в специальных классах функций // Проблемы оптимального управления.. Минск: Наука и техника 1981. С. 157-175.

[18] Silverman L. M., Meadows H. E. Controllability and observability in time-variable linear systems // SIAM J. Contr. 1967. V. 5. №1. P. 64-73.

[19] Chang A. An algebraic characterization of controllability // IEEE Trans. Autom. Contr. 1965. V. 10. № 5. P. 112-113.

[20] Гайшун И. В., Астровский А. И. Описание множества равномерно наблюдаемых линейных нестационарных систем // Докл. АН Беларуси. 1996. Т. 40. № 5. С. 5-8.

[21] Weiss L. The concepts of differential controllability and differential observability // J. Math. Anal. Appl. 1965. №10. P. 442-449. 